\title{
Probing the Atomic Lattice Response of Quantum Materials Across Phase Transitions
}

Lena F. Kourkoutis ${ }^{1,2}$, Ismail El Baggari ${ }^{3}$, Benjamin H. Savitzky ${ }^{3}$, David J. Baek ${ }^{4}$, Berit H. Goodge ${ }^{1}$, Michael J. Zachman ${ }^{1}$

1. School of Applied and Engineering Physics, Cornell University, Ithaca, NY, USA.

2. Kavli Institute at Cornell, Cornell University, Ithaca, NY, USA.

3. Department of Physics, Cornell University, Ithaca, NY, USA.

4. School of Electrical and Computer Engineering, Cornell University, Ithaca, NY, USA.

Coupling between charge, spin, lattice, or orbital degrees of freedom gives rise to remarkable phenomena such as colossal magnetoresistance, metal-insulator transitions and high temperature superconductivity. Many aspects of these competing interactions, however, remain elusive. Traditional bulk measurements of phases transitions, which average the material's response over large length scales, can inferred the presences of spatial inhomogeneity. Understanding their character, however, requires local probes. Direct, real-space measurements of the onset of emerging order are key to understanding the diverse properties of complex electronic materials and to ultimately harness them for applications.

Today, sub-Ångstrom resolution imaging is routinely achieved by aberration-corrected scanning transmission electron microscopy (STEM). In addition to resolving individual atomic columns in crystals, STEM can be used to determine their positions with picometer precision [1]. This has enabled direct mapping of functional atomic displacements in ferroelectric materials, visualization of polar vortices in oxide multilayers and periodic lattice displacements (PLDs) of room-temperature charge-ordered phases $[2,3,4]$. So far, the majority of atomic-resolution STEM measurements have been performed at room temperature, where stage stability is high, a requisite for acquisition of high resolution, high signal-tonoise data. However, extending the reach of high-resolution STEM to cryogenic temperatures promises access to low temperature phases including high temperature superconductivity, charge density waves, multiferroics and metal-insulator transitions.

Here, we demonstrate recent advances in aberration-corrected cryo-STEM using a liquid nitrogen $\left(\mathrm{LN}_{2}\right)$ double-tilt side-entry holder (Gatan Model 626) and a customized FEI Titan Themis equipped with a cryogenically cooled box which encloses the sample during low temperature imaging to reduce ice buildup. By combining cryogenic cooling, rapid image acquisition and optimized data processing, we map lattice displacements in a manganite at $\mathrm{LN}_{2}$ temperature, well below the charge-order transition temperature. We find that cations undergo picometer-scale displacements (Fig. 1) and that models based solely on separation of charge or orbital ordering are insufficient. Such atomic displacements change bond distances, bond angles, and hence exchange interactions, and may be key to explaining how charge order impacts other electronic states. We further directly visualize temperature-dependent nanosocale inhomogeneity in the displacements which explain shifts in the modulation wave vector previously observed in bulk measurements (Fig. 2). Aberration-corrected cryo-STEM opens a path to quantify the role of the lattice in low temperature electronic phases with high resolution and precision [5].

References:

[1] A. B. Yankovich et al., Nature Communications 5 (2014), p. 4155.

[2] J. A. Mundy et al., Nature 537 (2016), p. 523. 
[3] A. K. Yadav et al., Nature 530 (2016), p. 198.

[4] B. H. Savitzky, I. E. Baggari et al., Nature Communications 8 (2017), p. 1883.

[4] I. E. Baggari et al., PNAS Latest Articles (2018), 201714901.

[5] Work supported by AFOSR (FA 9550-16-1-0305), NSF (DMR-1539918, DMR-1429155), and the Packard Foundation. This work made use of CCMR shared facilities supported by NSF (DMR-1719875).
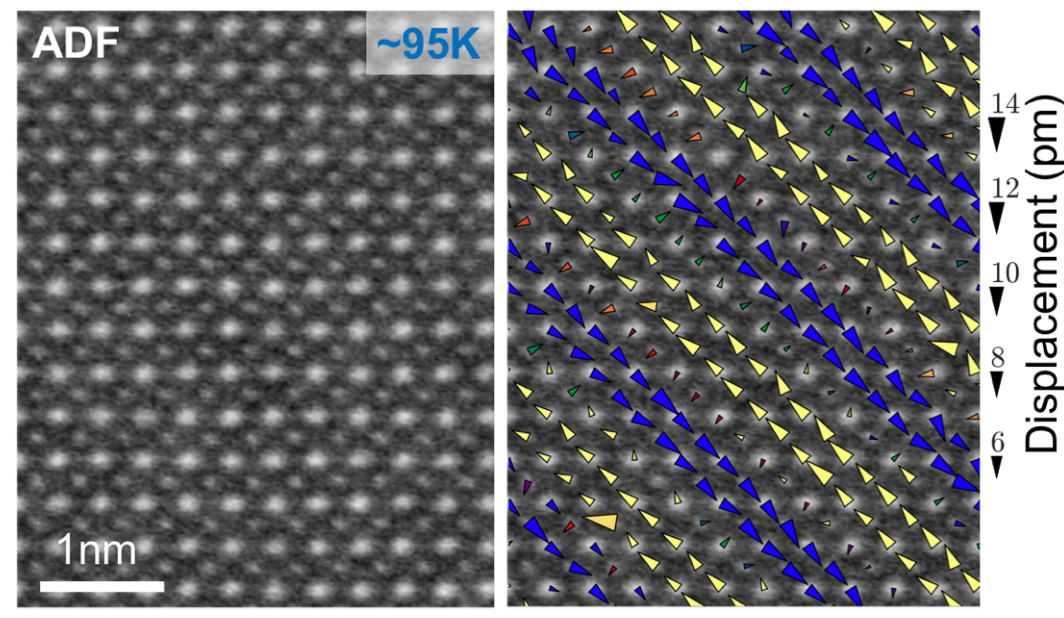

Figure 1. Aberration-corrected cryoSTEM imaging of a charge-ordered manganite $(\mathrm{Bi}, \mathrm{Sr}, \mathrm{Ca}) \mathrm{MnO}_{3}$ performed at cryogenic temperature $(\sim 95 \mathrm{~K})$. Image was obtained by rigid registration of a series of HAADF STEM images recorded at a dwell time of $0.5 \mu \mathrm{s} /$ pixel. (right) Overlaid arrows map picometerscale periodic displacements of atomic columns associated with charge ordering. Area of arrows scales linearly with the magnitude of the displacement.
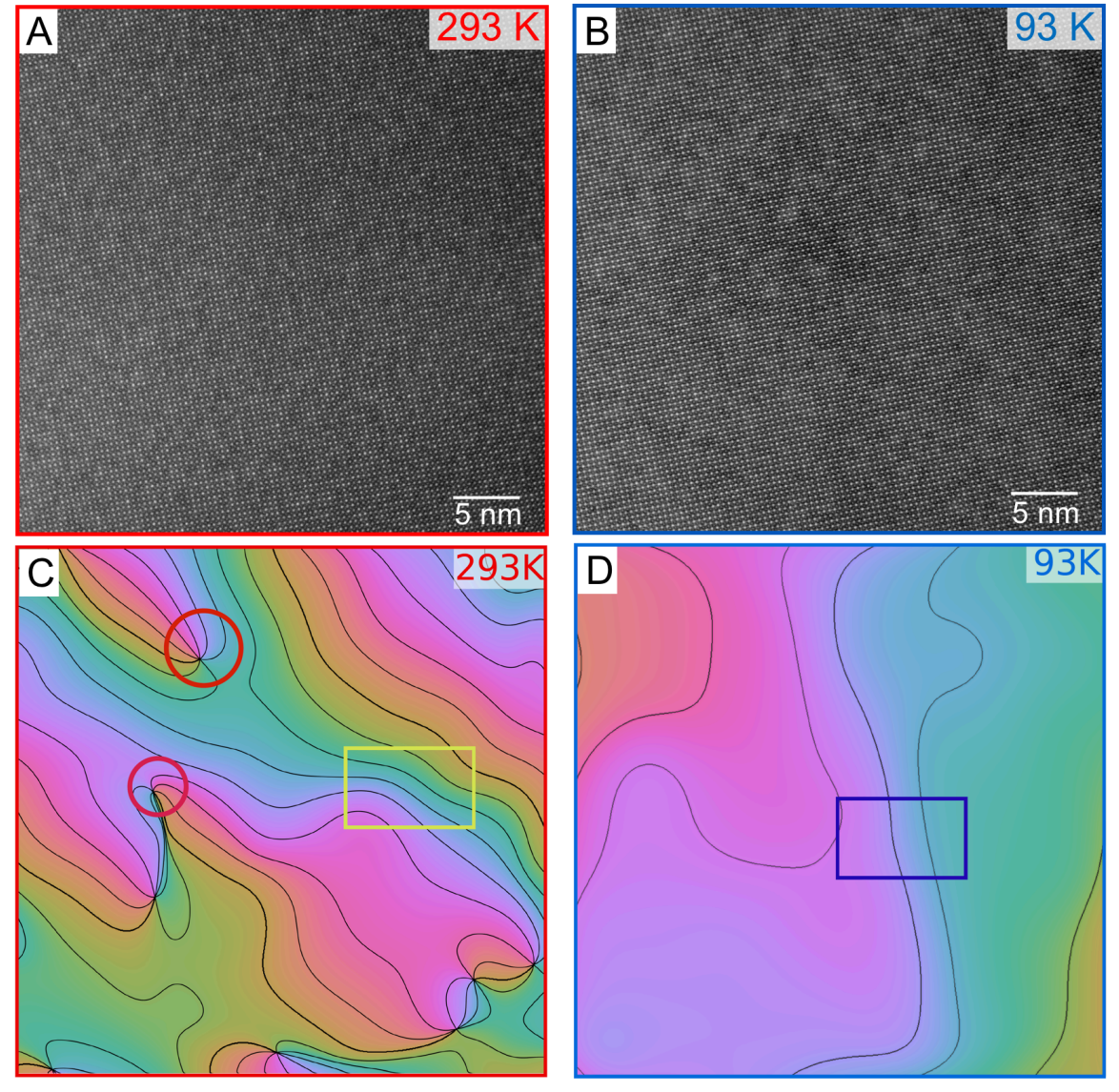

0

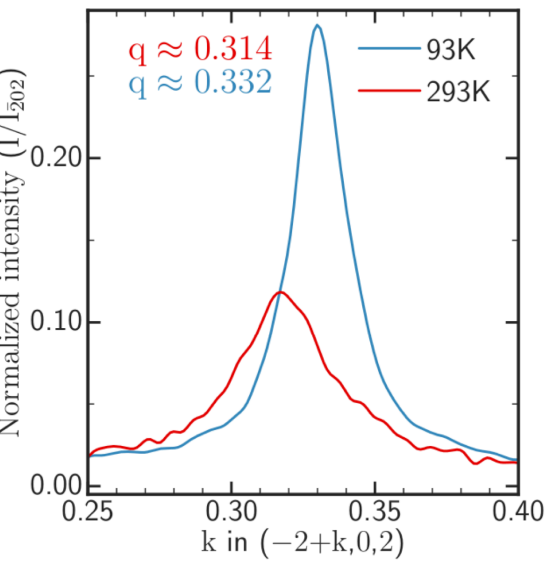

Figure 2. (A, B) HAADF STEM imaging of the same manganite sample at room and cryogenic temperatures. (C, D) Coarse grained phase of the lattice displacements shows local disorder at room temperature and emergence of phase coherence at $93 \mathrm{~K}$ far below the $\mathrm{T}_{\mathrm{c}}$ of $\sim 320 \mathrm{~K}$. Black lines in $\mathrm{C}$ and $\mathrm{D}$ represent constant $\pi / 4$ phase contours. Red circles correspond to dislocations in the PLDs, and boxes correspond to shear deformations. Phase gradients give rise to expansion and compression of wave fronts and result in wave vector shifts in reciprocal space as measured by diffraction (top right). 